\title{
FUNCTIONAL COMPOSITION ON SOBOLEV SPACES
}

\author{
BY MOSHE MARCUS AND VICTOR J. MIZEL ${ }^{1}$ \\ Communicated by James Serrin, July 23, 1971
}

Introduction. This announcement concerns the study of conditions on functions $g\left(x, t_{1}, \ldots, t_{m}\right), x \in \Omega \subset R_{n}$, under which the function $g$ provides, via composition, a mapping from certain product Sobolev spaces $W_{r, q}(\Omega)^{m}$ to other Sobolev spaces $W_{1, p}(\Omega)$. Such questions are of interest in the study of nonlinear partial differential equations and elsewhere (see [4], for example). Much of the analysis hinges on the use of a (seemingly new) notion of absolute continuity on tracks of absolutely continuous curves. This notion may also be useful elsewhere.

Statement of results. In what follows $\mathscr{H}_{1}$ denotes one-dimensional Hausdorff measure, $\mathscr{L}_{n}$ denotes $n$-dimensional Lebesgue measure, and $R_{k}$ denotes $k$-dimensional Euclidean space.

Let $T \subset R_{m}$ be the track of an absolutely continuous curve. Then by results of Roger [5] and Federer [1], for all points $y \in T$ with the exception of an $\mathscr{H}_{1}$-null set there is a unique pair of tangent directions to $T$ at $\boldsymbol{y}$, to be denoted by unit vectors $\theta_{y},-\theta_{y}$. Let $g: T \rightarrow R_{1}$ be defined on $T$. We say that $g$ has a tangential derivative at $y$ if, at $y, T$ possesses a unique pair of tangent directions and if both the following relations hold for sequences $\left\{\boldsymbol{y}_{i}\right\} \in T$ :

$$
\begin{aligned}
\frac{\overline{y y_{i}}}{\left|y_{i}-y\right|} \rightarrow \theta_{y} & \Rightarrow \frac{g\left(y_{i}\right)-g(y)}{\left|y_{i}-y\right|} \rightarrow \Lambda, \\
\frac{\overline{y y_{i}}}{\left|y_{i}-y\right|} \rightarrow-\theta_{y} & \Rightarrow \frac{g\left(y_{i}\right)-g(y)}{\left|y_{i}-y\right|} \rightarrow-\Lambda, \quad \Lambda \in R_{1} .
\end{aligned}
$$

In this case the quantity $|\Lambda| \equiv D_{T} g(y)$ is called the tangential derivative of $g$ at $y$. We shall say that $g$ is absolutely continuous on $T$ provided that $D_{T} g$ is defined $\mathscr{H}_{1}$-a.e. on $T$ (in this case $D_{T} g$ is necessarily $\mathscr{H}_{1}$-measurable) and the following relation is satisfied

$$
\left|g(y)-g\left(y^{\prime}\right)\right| \leqq \int_{U} D_{T} g(y) d \mathscr{H}_{1}<\infty
$$

whenever $y, y^{\prime} \in T$ and $U \subset T$ is a closed connected subset containing $y$ and $\boldsymbol{y}^{\prime}$. Call a function $g: R_{m} \rightarrow R_{1}$ fully absolutely continuous provided

AMS 1969 subject classifications. Primary 3538, 2880; Secondary 4638.

Key words and phrases. Sobolev spaces, absolute continuity on tracks, full absolute continuity, chain rule, total differential, cone property, locally Lipschitz.

${ }^{1}$ Partially supported by NSF Grants GP 24339 and GP 28377. 
that it is a Borel function and is absolutely continuous on all such tracks $T$.

The above notion of absolute continuity on a track coincides with the usual one when $m=1$ and $T$ is a real interval, and for arbitrary $m$ shares many of the properties of the notion of absolute continuity for real functions on an interval. In particular, every locally Lipschitz function on $R_{m}$ can be shown to be fully absolutely continuous. Moreover the following generalization of a classical chain rule of Vallée Poussin is available.

THEOREM 1. Suppose that $v=\left(v_{1}, \ldots, v_{m}\right): I \rightarrow R_{m}$ is absolutely continuous on the interval I and that $g: R_{m} \rightarrow R_{1}$ has a total differential at all points of the track $T=v(I)$ except for an $\mathscr{H}_{1}$-null set, and is such that $\left.g\right|_{T}$ is absolutely continuous on $T$. Then the composite function $w=g\left(v_{1}, \ldots, v_{m}\right): I \rightarrow R_{1}$ is absolutely continuous if and only if the following function is Lebesgue integrable on I:

$$
w_{1}=\sum \frac{\partial g}{\partial t_{j}}(v) \dot{v}_{j},
$$

(all products being interpreted as zero wherever their second factor is zero). In that case

$$
\dot{w}=w_{1} \text { almost everywhere on } I \text {. }
$$

Combining this result with a characterization, due to Gagliardo [2], of the Sobolev spaces $W_{1, q}(\Omega)$ (where $W_{1, q}(\Omega)$ is the class of $L_{q}$ functions with $L_{q}$ strong first derivatives on the domain $\Omega \subset R_{n}$ ), we obtain the following results.

THeOREM 2. Let $\Omega$ be a domain in $R_{n}$. Suppose that $g: R_{m} \rightarrow R_{1}$ is fully absolutely continuous and that its points of nondifferentiability meet the track of every absolutely continuous curve in an $\mathscr{H}_{1}$-null set. Let $\boldsymbol{u}=\left(u_{1}, \ldots, u_{m}\right)$ where $u_{j} \in W_{1,1}^{\text {loc }}(\Omega), 1 \leqq j \leqq m$, and set $v=g\left(u_{1}, \ldots, u_{m}\right)$. Then $v \in W_{1,1}^{\text {loc }}(\Omega)$ if and only if the functions

$$
v_{i}=\sum_{j=1}^{m} \frac{\partial g}{\partial t_{j}}(u) \partial_{i} u_{j}, \quad i=1, \ldots, n,
$$

belong to $L_{1}^{\mathrm{loc}}(\Omega)$, where $\partial_{i}$ denotes strong differentiation and where the products are to be interpreted as zero wherever their second factor is zero. In that case

$$
v_{i}=\partial_{i} v \quad \mathscr{L}_{n} \text {-a.e. in } \Omega, \quad i=1, \ldots, n .
$$

For the case $m=1$, i.e. $g: R_{1} \rightarrow R_{1}$ locally absolutely continuous in the usual sense, this result was obtained by Serrin [6]. Serrin's result stimulated our interest in obtaining the results stated above under assumptions which generalize the usual notion of absolute continuity. 
REMARK. The need for some hypothesis on the nondifferentiability points of $g$ is clear from the following example with $n=1$. Let $g\left(t_{1}, \ldots, t_{m}\right)$ $=\max \left(t_{1}, \ldots, t_{m}\right)$ and let $u_{1}(x)=\cdots=u_{m}(x)=x, \forall x \in(0,1)$. Then the function $v_{1}$ is nowhere defined on $\Omega=(0,1)$, while $\partial_{1} v \equiv 1$.

On the other hand it is possible for functions $g$ to provide mappings between Sobolev spaces even in the absence of chain rules. This aspect of the problem is studied in [3].

THEOREM 3. Let $\Omega$ be a bounded domain in $R_{n}$ possessing the cone property. Let $g$ be a function satisfying the assumptions in Theorem 2 and denote $h_{j}=\partial g / \partial t_{j} \quad\left(h_{j}\right.$ is necessarily Borel measurable and defined $\mathscr{L}_{n}$-a.e.), $j=1, \ldots, m$. Given $p, 1 \leqq p \leqq n$, suppose that for some $q, p<q<n$, the functions $h_{j}$ determine, via composition, mappings which satisfy:

$$
h_{j}: L_{q^{*}}(\Omega)^{m} \rightarrow L_{q^{\prime}}(\Omega) \quad(j=1, \ldots, m), \quad \text { with } q^{*}=\frac{n q}{n-q}, q^{\prime}=\frac{p q}{q-p} .
$$

Then $g$ yields, via composition, a mapping which satisfies:

$$
g: W_{1, q}(\Omega)^{m} \rightarrow W_{1, p}(\Omega)
$$

Moreover, with $v=g\left(u_{1}, \ldots, u_{m}\right)$ one has for $\boldsymbol{u}=\left(u_{1}, \ldots, u_{m}\right) \in W_{1, q}(\Omega)^{m}$

$$
\partial_{i} v=\sum_{j=1}^{m} \frac{\partial g}{\partial t_{j}}(\boldsymbol{u}) \partial_{i} u_{j}, \quad i=1, \ldots, n,
$$

the products being interpreted as zero wherever their second factor is zero.

REMARK. Such conditions on the $h_{j}$ hold in particular when the $h_{j}$ satisfy growth conditions of the following sort:

$$
\left|h_{j}(\boldsymbol{t})\right| \leqq a+b|\boldsymbol{t}|^{\alpha}, \quad \alpha=q^{*} / q^{\prime}, 1 \leqq j \leqq n .
$$

We have the following type of result for higher order Sobolev spaces.

THEOREM 4. Let $\Omega$ be a domain in $R_{n}$. Suppose that $g: R_{n+1} \rightarrow R_{1}$ is fully absolutely continuous and that its points of nondifferentiability meet the track of every absolutely continuous curve in a set whose projection onto the $t_{0}$-axis in $R_{n+1}$ is a Lebesgue null set. Let $u \in W_{2,1}^{\text {loc }}(\Omega)$ and set $v=$ $g\left(u, \partial_{1} u, \ldots, \partial_{n} u\right)$. Then $v \in W_{1,1}^{\text {loc }}(\Omega)$ if and only if the functions

$$
v_{i}=\frac{\partial g}{\partial t_{0}}(\boldsymbol{u}) \partial_{i} u+\sum_{j=1}^{n} \frac{\partial g}{\partial t_{j}}(\boldsymbol{u}) \partial_{i} \partial_{j} u, \quad i=1, \ldots, n,
$$

belong to $L_{1}^{\text {loc }}(\Omega)$, where the products are to be interpreted as zero wherever their second factor is zero. In that case we have

$$
v_{i}=\partial_{i} v \quad \mathscr{L}_{n} \text {-a.e. in } \Omega, \quad i=1, \ldots, n .
$$

This can be exploited to obtain conditions on the functions $h_{j}=\partial g / \partial t_{j}$, 
$0 \leqq j \leqq n$, as mappings between spaces $L_{\rho}(\Omega)$, which ensure that $g$ yields, via composition, a mapping of Sobolev spaces of the following type:

$$
g: W_{2, q}(\Omega) \rightarrow W_{1, p}(\Omega), \quad 1 \leqq p<q \leqq n / 2 .
$$

The results, which are analogues of Theorem 3 are omitted here.

We sketch the proof of Theorem 2. By Gagliardo's result [2] we are able to restrict attention to functions $u_{j}$ which are locally absolutely continuous (as a function of one variable) on $\tau \cap \Omega$ for almost all lines $\tau$ parallel to any axis $x_{i}$ in $R_{n}$. We may then utilize Theorem 1 to show that $v$ is absolutely continuous on subintervals of $\tau \cap \Omega$, when and only when $\partial v / \partial x_{i}$ coincides almost everywhere with $v_{i}$ on such subintervals. We then utilize Gagliardo's characterization, once more, to deduce that $v \in W_{1,1}^{\text {loc }}(\Omega)$ if and only if $v_{i} \in L_{1}^{\text {loc }}(\Omega), 1 \leqq i \leqq n$.

For functions $g$ defined on $\Omega \times R_{m}$ we have closely analogous results to the above provided that $g$ is locally Lipschitz (rather than merely fully absolutely continuous) on $\Omega \times R_{m}$. As a sample result of this type we mention the following.

THEOREM 5. Let $\Omega$ be a bounded domain in $R_{n}$ possessing the cone property. Suppose that $g: \Omega \times R_{m} \rightarrow R_{1}$ is locally Lipschitz on $\Omega \times R_{m}$. Suppose also that the set of nondifferentiability points of $g$ has the property that its projection on $R_{m}$ meets the track of every absolutely continuous curve in $R_{m}$ in an $\mathscr{H}_{1}$-null set and, in addition, that for all $(\boldsymbol{x}, \boldsymbol{t}) \in \Omega \times R_{m}$ where the derivatives mentioned below exist, they satisfy the growth conditions:

$$
\begin{array}{ll}
\left|\frac{\partial g}{\partial x_{i}}(\boldsymbol{x}, \boldsymbol{t})\right| \leqq a_{1}(\boldsymbol{x})+b_{1}|\boldsymbol{t}|^{\nu}, & i=1, \ldots, n, \\
\left|\frac{\partial g}{\partial t_{j}}(\boldsymbol{x}, \boldsymbol{t})\right| \leqq a_{2}(\boldsymbol{x})+b_{2}|\boldsymbol{t}|^{\nu-1}, & j=1, \ldots, m,
\end{array}
$$

where $v \geqq 1$ is a fixed number; $a_{1} \in L_{p}(\Omega)$ for some $1<p<n ; a_{2} \in L_{r}(\Omega)$ with $r=(p v /(v-1)) \cdot(n /(n-p))[n=\infty$ for $v=1]$; and $|\boldsymbol{t}|=\left|t_{1}\right|+\cdots$ $+\left|t_{m}\right|$. Then $g$ yields, via composition, a mapping of a Sobolev space $W_{1, q}(\Omega)^{m}$ into $W_{1, p}(\Omega)$ :

$$
g: W_{1, q}(\Omega)^{m} \rightarrow W_{1, p}(\Omega) \text { with } q=v p \cdot n /(n+(v-1) p),
$$

and for all $\boldsymbol{u}=\left(u_{1}, \ldots, u_{m}\right) \in W_{1, q}(\Omega)^{m}$,

$$
\left\|g\left(x, u_{1}(x), \ldots, u_{m}(x)\right)\right\|_{W_{1, p}(\Omega)} \leqq \operatorname{const}\left[1+\left\|\left(u_{1}, \ldots, u_{m}\right)\right\|_{W_{1, q}(\Omega)^{m}}^{v}\right],
$$

where the constant depends on $\Omega, a_{1}, a_{2}, b_{1}, b_{2}$ and $g_{0}(x) \equiv g(x, 0, \ldots, 0)$, but not on $\boldsymbol{u}$. Moreover this mapping is demicontinuous, i.e. continuous from the strong to the weak topology.

Proofs and related results will appear elsewhere. 


\section{BIBLIOGRAPHY}

1. H. Federer, Geometric measure theory, Die Grundlehren der math. Wissenschaften, Band 153, Springer-Verlag, New York, 1969. MR 41 \# 1976.

2. E. Gagliardo, Proprietà di alcune classi di funzioni in più variabili, Ricerche Mat. 7 (1958), 102-137. MR 21 \# 1526

3. M. Marcus and V. J. Mizel, Nemitsky operators on Sobolev spaces, CMU Technical Report, July 1971.

4. J. Moser, A rapidly convergent iteration method and non-linear partial differential equations. I, Ann. Scuola Norm. Sup. Pisa (3) 20 (1966), 265-315. MR 33 \# 7667.

5. F. Roger, Sur la relation entre les propriétés tangentielles et métriques des ensembles cartesiens, C. R. Acad. Sci. Paris 201 (1935), 871-872.

6. J. Serrin, Personal communication.

Department of Mathematics, Carnegie-Mellon University, Pittsburgh, PennsylVANIA 15213

Current address: (M. Marcus) Department of Mathematics, Technion, Haifa, Israel 